\title{
Continuous Cohomology of Permutation Groups on Profinite Modules
}

\author{
D M Evans and P R Hewitt
}

\begin{abstract}
Model theorists have made use of low-dimensional continuous cohomology of infinite permutation groups on profinite modules: see $[\mathbf{1}, \mathbf{6}, \mathbf{7}, \mathbf{9}]$, for example. We expand the module category in order to widen the cohomological toolkit. For an important class of groups we use these tools to establish criteria for finiteness of cohomology.

Mathematics Subject Classification (2000): 20B27, 22E41, 03C99.
\end{abstract}

\section{Introduction}

The symmetric group on any set carries a natural topology in which the pointwise stabilizers of finite sets form a base of open neighbourhoods of the identity. Permutation groups on the set are then considered as topological groups with the subspace topology, and a permutation group is closed in the symmetric group if and only if it is the automorphism group of some collection of relations on the set. Conversely, if $G$ is a Hausdorff group whose topology is generated by a family $U_{i}$ of open subgroups then $G$ is isomorphic (as a topological group) to a permutation group acting on the disjoint union of the coset spaces $G / U_{i}$. We denote the category of permutation groups regarded as topological groups, together with continuous homomorphisms by $\mathcal{P} \mathcal{G}$.

A permutation group on a set $\Omega$ is compact if and only if it is closed in the symmetric group on $\Omega$ and all of its orbits are finite: thus compact permutation groups are closed subgroups of direct products of finite groups, and so are exactly the profinite groups. Consider a short exact sequence in $\mathcal{P G}$

$$
1 \rightarrow K \rightarrow \underset{1}{E} \rightarrow G \rightarrow 1
$$


where $K$ is compact and abelian. Then $K$ is naturally a $G$-module and the action $G \times K \rightarrow K$ is continuous. If $G \in \mathcal{P G}$ then we denote by $\mathbf{P}_{G}$ the abelian category of continuous, profinite $G$-modules and continuous $G$-homomorphisms. It is worth noting that Pontryagin duality implies that the dual category $\widehat{\mathbf{P}_{G}}$ can be thought of as the category of continuous, discrete torsion $G$-modules and $G$-homomorphisms.

A well-known argument (cf. [11], Proposition I.1) shows that continuous epimorphisms in $\mathbf{P}_{G}$ admit sections which are continuous functions. A slight extension of this argument (which we omit) shows that a short exact sequence (1) admits a continuous closed section $G \rightarrow E$, and so the cohomology of low-degree continuous cocycles retains its familiar applications: $\mathrm{H}_{c}^{1}(G, K)$ classifies closed complements in the split extension and $\mathrm{H}_{c}^{2}(G, K)$ classifies all extensions in $\mathcal{P} \mathcal{G}$.

The problem of classifying extensions as in (1) has occurred in model theory. We refer the reader to $([\mathbf{6}]$, Section 1) for a concise introduction to this, and [7] for a fuller survey. Of particular relevance for the model theory is the computation of $\mathrm{H}_{c}^{1}(G, M)$ where $G$ is the automorphism group of some suitably nice first-order structure and $M$ is a quotient of a permutation module $A^{\Omega}$. By the latter, we mean that $\Omega$ is a transitive $G$-space with the stabilizer $H$ of some point being open, and $A$ is a finite abelian group. (More formally, $A^{\Omega}$ is a coinduced module $A \Uparrow_{H}^{G}$ : see the beginning of Sections 2 and 3.) We denote by $\mathbf{F}_{G}$ the subcategory of $\mathbf{P}_{G}$ consisting of closed submodules of finite direct sums of such permutation modules: these are precisely the elements of $\mathbf{P}_{G}$ whose Pontryagin duals are finitely generated as $G$ modules. In general $\mathbf{F}_{G}$ need not be closed under quotients. However, for certain important infinite permutation groups $G$ (in particular, the countable dimensional classical groups over finite fields) it is known that modules in $\mathbf{F}_{G}$ satisfy the descending chain condition on closed submodules, and it then follows that $\mathbf{F}_{G}$ is closed under quotients and so is an abelian category.

In this paper we develop systematically various results for the cohomology groups $\mathrm{H}_{c}^{n}(G, K)$ determined by continuous cochains (for $G \in \mathcal{P G}$ and $K \in \mathbf{P}_{G}$ ): something which was done in a very ad hoc fashion in [7] for the case $n=1$. Because of the existence of continuous sections for epimorphisms between profinite groups, we already have the long exact sequence of cohomology. More awkward (because it is not clear whether $\mathbf{P}_{G}$ has enough projectives/injectives) is Shapiro's Lemma, but we give a direct argument for this in Proposition 2.2. The principal application of these results is a useful permutation group theoretic criterion on $G$ (Theorem 3.5) which implies that the finite trivial 
modules $A$ in $\mathbf{P}_{G}$ are acyclic (that is, $\mathrm{H}_{c}^{n}(G, A)$ is zero for $n \geq 1$ ). Using this, we deduce (Corollary 3.6) that for certain infinite permutation groups $G$, the categories $\mathbf{P}_{G}$ and $\mathbf{F}_{G}$ have enough acyclics and the continuous cohomology groups $\mathrm{H}_{c}^{n}(G, K)$ are finite for $K \in \mathbf{F}_{G}$.

As a technical intermediate step in deriving this criterion, we show that certain direct limits of permutation modules are 'acyclic.' However, such limits are not profinite groups, but weakly profinite: they have the weak topology determined by a countable family of profinite subgroups. In Section 1 we develop some basic results about such groups (in particular, we show that they are topological groups: Proposition 1.5) and in Section 2 we extend the continuous cohomology on $\mathbf{P}_{G}$ to 'bounded' cohomology on these weakly profinite modules.

\section{Weakly profinite groups}

We say that a group $M$ equipped with a topology is weakly profinite if it is the union of a countable increasing chain $\left\{M_{i}: i \in \mathbb{N}\right\}$ of profinite subgroups with respect to which it carries the weak topology. Thus, a subset of $M$ is open precisely when its intersection with each $M_{i}$ is open. Equivalently, a map from $M$ into any space is continuous when its restriction to each $M_{i}$ is continuous. We refer to $\left\{M_{i}\right\}$ as a profinite system for $M$.

It is not clear whether or not a weakly profinite group is a topological group, although we can establish this for abelian groups. However, since both the algebra and topology on $M$ are determined by their restrictions to the $M_{i}$, we see that translation by any element of $M$ is a homeomorphism of $M$.

Proposition 1.1. Suppose $M$ is weakly profinite with respect to $\left\{M_{i}\right\}$. If $K$ is a compact subset of $M$, then $K$ is contained in one of the $M_{i}$.

Proof. If not then $K \cap\left(M_{i+1} \backslash M_{i}\right)$ is non-empty for infinitely many $i$. If $X$ contains one element of this for each such $i$, then $X$ is an infinite subset of $K$ whose intersection with each $M_{i}$ is finite. It follows that $X$ is a closed, discrete subset of $K$, which is impossible.

A subset of $M$ is bounded if it is contained in some $M_{i}$. The proposition says that compacta are bounded, so bounded subsets are those whose closure is compact. Similarly a map to $M$ is bounded when its image is contained in a compact subset.

Proposition 1.2. If $M$ is a weakly profinite group and $N$ is a closed subgroup then both $N$ and $M / N$ are weakly profinite. 
ProOF. Suppose $M$ is weakly profinite with respect to a family $\left\{M_{i}\right\}$. It is straightforward to show that the relative topology on $N$ is the weak topology with respect to the $N \cap M_{i}$, and that the quotient topology on $M / N$ is the weak topology with respect to the $p\left(M_{i}\right)$, where $p$ is the quotient map. We consider the latter in detail.

Suppose $\bar{X}$ is a subset of $M / N$ with the property that each $\bar{X} \cap$ $p\left(M_{i}\right)$ is open in $p\left(M_{i}\right)$. Now

$$
p^{-1}(\bar{X}) \cap M_{i}=p^{-1}\left(\bar{X} \cap p\left(M_{i}\right)\right) \cap M_{i} .
$$

Since $p$ is continuous this intersection is open in $M_{i}$. As $M$ has the weak topology we conclude that $p^{-1}(\bar{X})$ is open. Hence $\bar{X}$ is open, by definition of the quotient topology.

Proposition 1.3. If $\theta: M \rightarrow L$ is a continuous epimorphism between weakly profinite groups then $\theta$ is an open map. In particular, if $\theta$ is an isomorphism then it is a homeomorphism.

Proof. The induced map $M / \operatorname{ker} \theta \rightarrow L$ is continuous, so by Proposition 1.2 it suffices to prove the final statement. Assume $\theta$ is a continuous isomorphism. As $L$ has the weak topology with respect to a family of profinite subgroups it suffices to show that $\theta^{-1}$ is continuous when restricted to one of these. The image of this is closed in $M$ so again we reduce to the case where $L$ is profinite.

If $M$ is also compact then the result is well-known. Otherwise $M$ is the union of a countable chain of proper compact subgroups $\left\{M_{i}\right\}$, necessarily of infinite index in $M$. Let $L_{i}=\theta\left(M_{i}\right)$. The $L_{i}$ are compact subgroups of $L$, also of infinite index in $L$, and hence nowhere dense. Since $L$ is compact this contradicts Baire's Theorem.

Propositions 1.1 and 1.3 tell us that the topology on a weakly profinite group $M$ is determined by any countable chain of compact subgroups whose union is $M$. Countability is crucial: an infinite power of a finite group is compact under the product topology but discrete under the weak topology generated by finite subgroups.

Let $M$ be the algebraic direct sum of a countable family of topological groups $M_{i}$. If we endow $M$ with the weak topology generated by finite direct sums of the $M_{i}$ then we obtain what is sometimes called the finite topology. In general the finite topology is not a group topology. Nevertheless the category of topological abelian groups does admit coproducts - the coproduct is some rather mysterious refinement of the finite topology. If the finite topology is a group topology then it is the coproduct topology. For more on this see [10]. The following is a special case of ([2], Corollary on p. 20). 
Proposition 1.4. Let $M=\oplus M_{i}$, with the finite topology. If the $M_{i}$ are compact then $M$ is a topological group.

Proposition 1.5. If $M$ is a weakly profinite abelian group then $M$ is a topological group.

Proof. Indeed, if $\left\{M_{i}\right\}$ is a profinite system for $M$ then since $M$ is abelian the canonical map $\oplus M_{i} \rightarrow M$ is a surjective, continuous, homomorphism. Now apply Propositions 1.2, 1.3, and 1.4.

Proposition 1.6. If $\phi: M \rightarrow N$ is a continuous surjective homomorphism of weakly profinite abelian groups then $\phi$ admits a closed, continuous section.

Proof. From Proposition 1.3 we know that $\phi$ is a quotient map and a profinite system for $N$ is obtained by taking the images of the groups in a profinite system for $M$. A section of $\phi$ is continuous if and only if its restriction to each of these compact subgroups of $N$ is continuous. Thus, to prove the proposition it is enough to observe that if $K \leq L$ are compact subgroups of $M$, then any continuous section of $\phi$ restricted to $K$ can be extended to a continuous section of $\phi$ restricted to $L$. But this is well-known: see ([11], Proposition I.1).

\section{Coinduced modules and bounded cohomology}

We work throughout with left modules and a Hausdorff topological group $G$. By a weakly profinite $G$-module $M$ we mean a weakly profinite abelian group with a profinite system of continuous $G$-submodules $M_{i}$. We do not require that the $G$-action be continuous globally, but only on compact submodules. A simple example should clarify the situation. Let $G$ be the symmetric group of countable degree and let $M$ be a countable direct sum of copies of $\mathbb{F}_{2}{ }^{\omega}$. The $G$-action on $M$ is not continuous, although it is continuous on each finite direct sum. The problem lies below the group action itself: in general the inclusion $A \times\left(\oplus B_{i}\right) \rightarrow \oplus\left(A \times B_{i}\right)$ need not be continuous.

If $G$ is a topological group, $H$ is a closed subgroup, and $M$ is a continuous $H$-module, then the coinduced module $M \Uparrow_{H}^{G}$ is the additive group of $H$-equivariant functions $f: G \rightarrow M$. Thus we require $f(x h)=$ $h^{-1} f(x)$ for all $h \in H$ and $x \in G$. The $G$-action is defined by the rule $(g f)(x)=f\left(g^{-1} x\right)$, for $g \in G$.

To describe the topology we can use the alternate description: $M \Uparrow_{H}^{G}$ is the space of sections of the $G$-bundle $G \times_{H} M \rightarrow G / H$, where $G \times_{H} M$ is the quotient of $G \times M$ by the diagonal $H$-action and $G / H$ is the left coset space. The topology on the coinduced module, regarded as a space of sections, is simply the compact-open topology. As noted 
above, in general the coinduced module is not a continuous $G$-module. However the action is continuous in one case of importance for us, namely when $H$ is open. In this case $G / H$ is discrete, and its compact subsets are finite. Hence $M \Uparrow_{H}^{G}$ is isomorphic, as a topological group, to the the product space $M^{G / H}$.

LEMMA 2.1. If $H$ is an open subgroup of $G$ and $M$ is a continuous $H$-module then the $G$-action on $M \Uparrow_{H}^{G}$ is continuous.

Proof. Let $N=M \Uparrow_{H}^{G}$. Suppose $x \in G, f \in N$, and $U$ is an open neighborhood of $(g f)(x)$. Choose open neighborhoods $V$ of 1 and $W$ of $(g f)(x)$ such that $V^{-1} W \subset U$. If $X=x(V \cap H) x^{-1} g$ and $Y=\left\{f^{\prime} \mid f^{\prime}\left(g^{-1} x\right) \in W\right\}$ then $X \times Y$ is an open neighborhood of $(g, f)$ such that $X Y \subset\left\{f^{\prime} \mid f^{\prime}(x) \in U\right\}$.

If $M$ is a continuous $G$-module consider the complex of continuous cochains $\left(\mathrm{C}_{c}^{n}(G, M) ; \delta^{n}\right)_{n \geq 0}$, where $\mathrm{C}_{c}^{n}(G, M)$ is the additive group of continuous functions $G^{n} \rightarrow M$, and $\delta^{n}: \mathrm{C}_{c}^{n}(G, M) \rightarrow \mathrm{C}_{c}^{n+1}(G, M)$ is the usual differential:

$$
\begin{gathered}
\delta^{n} f\left(x_{1}, \ldots, x_{n+1}\right)=x_{1} f\left(x_{2}, \ldots, x_{n+1}\right)+ \\
\sum_{i=1}^{n}(-1)^{i} f\left(x_{1}, \ldots, x_{i} x_{i+1}, \ldots, x_{n+1}\right)+(-1)^{n+1} f\left(x_{1}, \ldots, x_{n}\right) .
\end{gathered}
$$

Then the $n$-th continuous cohomology group $\mathrm{H}_{c}^{n}(G, M)$ is the kernel of $\delta^{n}$ modulo the image of $\delta^{n-1}$. We use similar notation for the cocomplex of continuous cocyles, and the subcomplexes of cochains and coboundaries. From the existence of continuous sections in $\mathbf{P}_{G}$ we deduce that any short exact sequence of profinite $G$-modules determines a short exact sequence of cochain complexes, and thence a long exact sequence in continuous cohomology.

Proposition 2.2 (Shapiro's Lemma). If $H$ is an open subgroup of the topological group $G$ and $M$ is a profinite $H$-module then $\mathrm{H}_{c}^{*}\left(G, M \Uparrow_{H}^{G}\right)$ is isomorphic to $\mathrm{H}_{c}^{*}(H, M)$.

Proof. There are various versions of this lemma in the literature. The version of the proof we sketch is essentially the same as in [8], whose target is locally compact groups. As there, we need merely highlight the points where the topology requires some care, since the formulas are the same as in the purely algebraic case.

The map $\psi: \mathrm{C}_{c}^{*}\left(G, M \Uparrow_{H}^{G}\right) \rightarrow \mathrm{C}_{c}^{*}(H, M)$ that induces an isomorphism in cohomology is, as usual, the combination of evaluation at 1 and restriction to $H$ :

$$
\psi(f)\left(x_{1}, \ldots, x_{n}\right)=f\left(x_{1}, \ldots, x_{n}\right)(1) .
$$


To define a right inverse to $\psi$ we need a retraction of $G$ onto $H$. Let $x \mapsto \tau_{x}$ be a transversal of the right coset space of $H$ in $G$. As this coset space is discrete, this map $G \rightarrow G$ is continuous. Define $r: G \rightarrow H$ as $r(x)=x \tau_{x}^{-1}$. This is a continuous retraction of $G$ onto $H$ with $r(h x)=h r(x)$ for all $h \in H$. Define

$$
\phi(f)\left(x_{1}, \ldots, x_{n}\right)(x)=r\left(\xi_{0}\right) \cdot f\left(r\left(\xi_{0}\right)^{-1} r\left(\xi_{1}\right), r\left(\xi_{1}\right)^{-1} r\left(\xi_{2}\right), \ldots\right),
$$

where $\xi_{i}=x^{-1} x_{1} \cdots x_{i}$. This map, defined for all cochains $f$, continuous or not, commutes with the differential. It is easy to see that $\phi(f)$ is continuous whenever $f$ is. Morever, $\psi \phi$ is the identity. To finish we show that $\phi \psi$ is homotopic to the identity. In fact, once again we can use the standard, algebraic chain homotopy $h$ given by:

$$
\begin{gathered}
(h g)\left(x_{1}, \ldots, x_{n}\right)(x)=g\left(x r\left(\xi_{0}\right), r\left(\xi_{0}\right)^{-1} r\left(\xi_{1}\right), r\left(\xi_{1}\right)^{-1} r\left(\xi_{2}\right), \ldots\right)(x) \\
-g\left(x_{1}, \xi_{1}^{-1} r\left(\xi_{1}\right), r\left(\xi_{1}\right)^{-1} r\left(\xi_{2}\right), r\left(\xi_{2}\right)^{-1} r\left(\xi_{3}\right), \ldots\right)(x) \\
+g\left(x_{1}, x_{2}, \xi_{2}^{-1} r\left(\xi_{2}\right), r\left(\xi_{2}\right)^{-1} r\left(\xi_{3}\right), r\left(\xi_{3}\right)^{-1} r\left(\xi_{4}\right), \ldots\right)(x) \ldots
\end{gathered}
$$

The standard formal (albeit tedious) computation shows that this is a chain homotopy. The easy lemma below (whose proof we omit) implies that this homotopy leaves the subcomplex of continuous cochains invariant.

LEMmA 2.3. If $H$ is open in $G$ and $f: G^{n} \rightarrow M \Uparrow_{H}^{G}$ is continuous, then $\hat{f}: G^{n+1} \rightarrow M$ defined by $\hat{f}\left(x_{0}, \ldots, x_{n}\right)=f\left(x_{1}, \ldots, x_{n}\right)\left(x_{0}\right)$ is continuous.

Suppose now that $M$ is a weakly profinite module for $G$. Let $\mathrm{H}_{b}^{*}(G, M)$ be the homology of the cochain complex of bounded, continuous cochains of $G$ in $M$ (with differential defined as above). We refer to this as the bounded cohomology of $G$. Note that if $M$ is profinite then $\mathrm{H}_{b}^{*}(G, M)=\mathrm{H}_{c}^{*}(G, M)$. Again, we use similar notation for the complex of bounded cocyles, and the subcomplexes of cochains and coboundaries.

As for continuous cohomology, the existence of sections (Proposition 1.6) implies that a short exact sequence of weakly profinite $G$ modules determines a long exact sequences in bounded cohomology.

If $\left\{M_{i}\right\}$ is a profinite system in $M$ then since direct limit commutes with homology we have that

$$
\mathrm{H}_{b}^{*}(G, M)=\underset{\lim }{\longrightarrow} \mathrm{H}_{c}^{*}\left(G, M_{i}\right) .
$$

Now suppose $G \in \mathcal{P} \mathcal{G}$ has a countable fundamental system of open subgroups (for example, $G$ is a permutation group on a countable set). If $M$ is a profinite $G$-module then define $M \Uparrow^{G}=\lim _{H} M \Uparrow_{H}^{G}$, where $H$ 
ranges over the countable fundamental system of open subgroups (of course, if $H_{1} \leq H_{2}$ are open subgroups of $G$, then $M \Uparrow_{H_{2}}^{G} \leq M \Uparrow_{H_{1}}^{G}$, so the directed set here is the reverse of the poset of open subgroups of $G$ ). In other words, $M \Uparrow{ }^{G}$ is the group of functions $G \rightarrow M$ each of which is equivariant with respect to some open subgroup. More generally, if $M$ is weakly profinite, say $M=\underline{\lim } M_{i}$, then define

$$
M \Uparrow^{G}=\underset{i}{\lim } M_{i} \Uparrow^{G}=\underset{i}{\underset{H}{\lim }} \underset{\lim _{i}}{\longrightarrow} M_{H}^{G} .
$$

If $M$ is a countable, torsion abelian group then the discrete topology on $M$ is the weak topology generated by all finite subgroups. By a trivial torsion module we mean such a group with trivial $G$-action.

Proposition 2.4. With the above notation, if $M$ is a trivial torsion $G$-module then $M \Uparrow^{G}$ is acyclic for bounded cohomology.

Proof. If $\left\{M_{i}\right\}$ is the system of finite subgroups then from equation 3 and Shapiro's Lemma we have that

$$
\mathrm{H}_{b}^{*}\left(G, M \Uparrow^{G}\right)=\underset{i}{\lim } \underset{H}{\lim } \mathrm{H}_{c}^{*}\left(H, M_{i}\right) .
$$

Suppose $f \in \mathrm{Z}_{c}^{*}\left(H, M_{i}\right)$. Without loss $f(1, \ldots, 1)=0$. Since $M_{i}$ is discrete $f$ is 0 when restricted to some open subgroup $L$. Hence the class of $f$ restricts to 0 in the limit.

One can also prove an injectivity property for $M \Uparrow^{G}$ when $M$ is a divisible torsion abelian group, but we will not need such a result.

\section{Acyclic resolutions via strong types}

In this section $G$ denotes a permutation group on an infinite set $\Omega$. We use weakly profinite modules to establish some permutationtheoretic criteria for finiteness and for vanishing of continuous cohomology. Throughout, $F$ denotes a finite abelian group regarded as a trivial $G$-module. We are interested initially in computing $\mathrm{H}_{c}^{*}(G, F)$.

By a continuous $G$-space $\Delta$ we mean one in which point stabilizers are open. We rephrase Proposition 2.4, which produces acyclic modules, in the language of transitive continuous $G$-spaces, and then generalize the result to intransitive $G$-spaces.

If the continuous $G$-space $\Delta$ is transitive, and $H$ is the stabilizer of a point $x$, then $F^{\Delta} \cong F \Uparrow_{H}^{G}$. Under this identification the Shapiro map from equation (2) becomes a weak equivalence - that is, an isomorphism in homology $\rho_{x}: \mathrm{Z}_{c}^{n}\left(G, F^{\Delta}\right) \sim \mathrm{Z}_{c}^{n}(H, F)$ with

$$
\rho_{x}(f)\left(g_{1}, \ldots, g_{n}\right)=f\left(g_{1}, \ldots, g_{n}\right)(x) .
$$


If $\pi: \Delta_{2} \rightarrow \Delta_{1}$ is a surjective $G$-map between transitive, continuous $G$-spaces and $\pi(y)=x$, then we have a commutative square

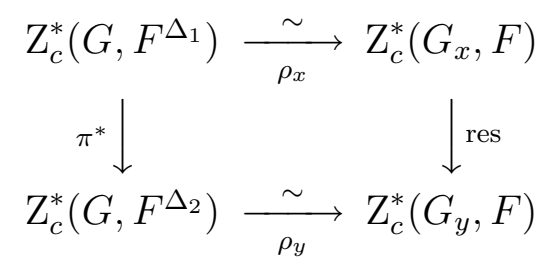

More generally, if $\Delta$ is any continuous $G$-space, with $G$-orbits $\left\{\Sigma_{i}\right.$ : $i \in I\}$, then $F^{\Delta}$ is the direct product of the $F^{\Sigma_{i}}$ and $\mathrm{H}_{c}^{*}\left(G, F^{\Delta}\right)$ is naturally isomorphic to the direct product of the $\mathrm{H}_{c}^{*}\left(G, F^{\Sigma_{i}}\right)$. So suppose $\pi: \Delta_{2} \rightarrow \Delta_{1}$ is a surjective $G$-map between continuous $G$-spaces. If $\{x\}$ and $\{y\}$ are systems of orbit representatives for $\Delta_{1}$ and $\Delta_{2}$, respectively, then $\{y\}$ is a lift of $\{x\}$ provided each $\pi(y) \in\{x\}$. For such a choice we have the commutative square

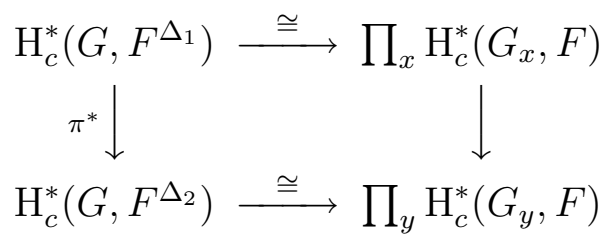

For an open subgroup $U$ of $G$ we say the lift is $U$-constrained provided each $G_{y}<U$. A tower $\cdots \stackrel{\pi_{3}}{\rightarrow} \Delta_{3} \stackrel{\pi_{2}}{\rightarrow} \Delta_{2} \stackrel{\pi_{1}}{\rightarrow} \Delta_{1}$ of continuous $G$-spaces with surjective $G$-maps is smooth if for every $\Delta_{i}$ there are orbit representatives $X_{i}$ with the property that for every open subgroup $U$ there is always a $U$-constrained lift of $X_{i}$ to some $\Delta_{j}$ (with $j>i$ ). The proof of Proposition 2.4 applies virtually without change under this hypothesis.

LEMMA 3.1. If $\left(\Delta_{i} ; \pi_{i}\right)$ is a smooth tower of continuous $G$-spaces, then $\lim _{\longrightarrow} F^{\Delta_{i}}$ is acyclic for bounded cohomology: $\mathrm{H}_{b}^{n}\left(G, \lim _{\longrightarrow} F^{\Delta_{i}}\right)=0$ if $n>0$.

We now give one way in which a tower of continuous $G$-spaces can be used to construct a resolution of $F$ by weakly profinite $G$-modules which are acyclic for bounded cohomology. Let $\Delta$ be a continuous $G$-space. Consider the cochain complex constructed from $n$-tuples $\Delta^{n}$ :

$$
\Sigma_{\Delta}: 0 \rightarrow F \rightarrow F^{\Delta} \stackrel{d_{1}}{\rightarrow} F^{\Delta^{2}} \stackrel{d_{2}}{\rightarrow} F^{\Delta^{3}} \stackrel{d_{3}}{\rightarrow} \cdots
$$

with $d_{n} f\left(a_{1}, \ldots, a_{n+1}\right)=\sum_{i}(-1)^{i} f\left(a_{1}, \ldots, \widehat{a_{i}}, \ldots, a_{n+1}\right)$ (where as usual the hat indicates omission). Clearly each $d_{n}$ is a continuous homomorphism of $G$-modules. Let $a^{*} \in \Delta$ and define $h_{n}: F^{\Delta^{n+1}} \rightarrow F^{\Delta^{n}}$ by $h_{n} f\left(a_{1}, \ldots, a_{n}\right)=f\left(a^{*}, a_{1}, \ldots, a_{n}\right)$. This is an abelian group homomorphism and $h_{n} d_{n}+d_{n-1} h_{n-1}$ is equal to minus the identity on $F^{\Delta^{n}}$. 
Thus $\Sigma_{\Delta}$ is contractible as a chain complex of abelian groups, and in particular, it is exact.

Now suppose that $T: \cdots \stackrel{\pi_{2}}{\rightarrow} \Delta_{2} \stackrel{\pi_{1}}{\rightarrow} \Delta_{1}$ is a tower of continuous $G$-spaces. Then we have a sequence of exact chain complexes $\Sigma_{\Delta_{1}} \rightarrow \Sigma_{\Delta_{2}} \rightarrow \ldots$ If we know that for each $j$ the tower $\left(\Delta_{i}^{j} ; \pi_{i}^{j}\right)$ is smooth, then each term $\lim _{i} F^{\Delta_{i}^{j}}$ of the direct limit is acyclic for $\mathrm{H}_{b}^{*}$, by Lemma 3.1. Since direct limits commute with homology we obtain a resolution of $F$ by acyclic modules:

$$
\Sigma_{T}: 0 \rightarrow F \rightarrow \underline{\lim _{\longrightarrow}} F^{\Delta_{i}} \rightarrow \underline{\lim _{\longrightarrow}} F^{\Delta_{i}^{2}} \rightarrow \underline{\lim _{\longrightarrow}} F^{\Delta_{i}^{3}} \rightarrow \cdots .
$$

We can then use $\Sigma_{T}$ to compute $\mathrm{H}_{c}^{*}(G, F)$ (which is the same thing as $\mathrm{H}_{b}^{*}(G, F)$ ): apply $\mathrm{H}_{b}^{0}$ to each term then take homology. Since $\mathrm{H}_{b}^{0}(G, M)$ is canonically the space of $G$-fixed points $M^{G}$, we find that

$$
\mathrm{H}_{c}^{*}(G, F)=H\left(\left(\mathrm{lim}_{\longrightarrow} F^{\Delta_{i}^{*}}\right)^{G}\right)=\varliminf_{\longrightarrow} H\left(\left(F^{\Delta_{i}^{*}}\right)^{G}\right) .
$$

We stress that equations (6) and (7) hold under the assumption that $\left(\Delta_{i}^{j} ; \pi_{i}^{j}\right)$ is a smooth tower, for each $j \in \mathbb{N}$ and remark that this does not follow automatically from the smoothness of $\left(\Delta_{i} ; \pi_{i}\right)$.

Suppose $\Delta$ is a continuous $G$-space. If $X \subseteq \Delta$, let $G_{X}$ denote the pointwise stabilizer of $X$. Consider a function $p$ which assigns to every finite $X \subseteq \Delta$ a $G_{X}$-orbit $p(X)$ on $\Delta \backslash X$. We say that $p$ is a strong type (over $\emptyset$ ) for $G$ on $\Delta$ if the following conditions are satisfied:

(1) If $X_{1} \subseteq X_{2}$ are finite then $p\left(X_{1}\right) \supseteq p\left(X_{2}\right)$.

(2) If $g \in G$ and $X$ is a finite subset of $\Delta$ then $g p(X)=p(g X)$. (This definition appeared as Definition 2.1 in [5], and the terminology originates in model theory. It would be more accurate to refer to $p$ as a type (over $\Delta$ ) which is non-splitting over the empty set.)

Suppose $p$ is a strong type for $G$ on $\Delta$. Let $\Delta^{(n)}$ consist of all $\left(a_{1}, \ldots, a_{n}\right) \in \Delta^{n}$ with $a_{1} \in p(\emptyset)$ and $a_{i+1} \in p\left(\left\{a_{1}, \ldots, a_{i}\right\}\right)$ : call these $p$-sequences (of length $n$ ). Note that $\Delta^{(n)}$ is a transitive, continuous $G$-space, and any subsequence of a $p$-sequence is also a $p$-sequence. Thus the differential $d_{i}$ defined previously also gives a continuous $G$ homomorphism from $F^{\Delta^{(i)}}$ to $F^{\Delta^{(i+1)}}$.

Lemma 3.2. Suppose $p$ is a strong type for $G$ on $\Delta$.

(1) The chain complex

$$
\left(F^{\Delta}\right)^{G} \stackrel{d_{1}}{\rightarrow}\left(F^{\Delta^{2}}\right)^{G} \stackrel{d_{2}}{\rightarrow}\left(F^{\Delta^{3}}\right)^{G} \stackrel{d_{3}}{\rightarrow} \cdots
$$

of $G$-fixed points is contractible (as a complex of abelian groups).

(2) The chain complex

$$
0 \rightarrow F \rightarrow F^{\Delta^{(1)}} \stackrel{d_{1}}{\rightarrow} F^{\Delta^{(2)}} \stackrel{d_{2}}{\rightarrow} F^{\Delta^{(3)}} \stackrel{d_{3}}{\rightarrow} \cdots
$$


is exact.

(3) The chain complex of G-fixed points

$$
0 \rightarrow F \rightarrow\left(F^{\Delta^{(1)}}\right)^{G} \stackrel{d_{1}}{\rightarrow}\left(F^{\Delta^{(2)}}\right)^{G} \stackrel{d_{2}}{\rightarrow}\left(F^{\Delta^{(3)}}\right)^{G} \stackrel{d_{3}}{\rightarrow} \cdots
$$

is exact.

Proof. (1) Note first that $D_{i}=\left(F^{\Delta^{i}}\right)^{G}$ is the set of maps which are constant on each $G$-orbit on $\Delta^{i}$. The contracting homotopy is defined in a similar way to the contracting homotopy $h$ for $\Sigma_{\Delta}$ given above, but to obtain the $G$-invariance, we use the strong type to choose $a^{*}$ rather than leaving it fixed.

Indeed, define $s_{i}: D_{i+1} \rightarrow D_{i}$ as follows. Let $f \in D_{i+1}$ and suppose $\left(x_{1}, \ldots, x_{i}\right) \in \Delta^{i}$. Choose $y$ to be any element of $p\left(\left\{x_{1}, \ldots, x_{i}\right\}\right)$, and define $\left(s_{i} f\right)\left(x_{1}, \ldots, x_{i}\right)=f\left(y, x_{1}, \ldots, x_{i}\right)$. As $p$ is a strong type and $f$ is $G$-invariant, one checks easily that $s_{i} f\left(x_{1}, \ldots, x_{i}\right)$ is independent of the choice of $y$, and that $s_{i} f$ is $G$-invariant. Now we claim that $s_{i} d_{i}+d_{i-1} s_{i-1}$ is equal to minus the identity map on $D_{i}$. Let $f \in D_{i}$. Then $\left(s_{i} d_{i} f\right)\left(x_{1}, \ldots, x_{i}\right)=\left(d_{i} f\right)\left(y, x_{1}, \ldots, x_{i}\right)$, where $y \in p\left(\left\{x_{1}, \ldots, x_{i}\right\}\right)$, and this is equal to

$$
-f\left(x_{1}, \ldots, x_{i}\right)+\sum_{j=1}^{i}(-1)^{j+1} f\left(y, x_{1}, \ldots, \widehat{x_{j}}, \ldots, x_{i}\right) .
$$

Now, $y \in p\left(\left\{x_{1}, \ldots, \widehat{x_{j}}, \ldots, x_{i}\right\}\right)$, by the first clause in the definition of a strong type. Thus

$$
\begin{gathered}
\left(d_{i-1} s_{i-1} f\right)\left(x_{1}, \ldots, x_{i}\right)=\sum_{j=1}^{i}(-1)^{j}\left(s_{i-1} f\right)\left(x_{1}, \ldots, \widehat{x}_{j}, \ldots, x_{i}\right) \\
=\sum_{j=1}^{i}(-1)^{j} f\left(y, x_{1}, \ldots, \widehat{x_{j}}, \ldots, x_{i}\right),
\end{gathered}
$$

whence the claim.

(2) Consider the Pontryagin dual of the complex, namely

$$
0 \longleftarrow F \stackrel{\eta}{\longleftarrow} F \Delta^{(1)} \stackrel{\delta_{1}}{\longleftarrow} F \Delta^{(2)} \stackrel{\delta_{2}}{\longleftarrow} \cdots .
$$

Here we have identified the finite abelian group $F$ with its dual, and $F \Delta^{(i)}$ is the free $F$-module with basis $\Delta^{(i)}$, with the obvious $G$-action. The $G$-homomorphisms $\eta$ and $\delta_{i}$ are given by the $F$-linear extensions of the maps $\eta(x)=1$ (for $x \in \Delta^{(1)}$ ) and

$$
\delta_{i}\left(x_{1}, x_{2}, \ldots, x_{i+1}\right)=\sum_{k}(-1)^{k}\left(x_{1}, \ldots, \hat{x}_{k}, \ldots, x_{i+1}\right),
$$


where $\left(x_{1}, \ldots, x_{i+1}\right) \in \Delta^{(i+1)}$. It will suffice to prove exactness of this dual complex.

Exactness at $F$ and $F \Delta^{(1)}$ is straightforward. For exactness at $F \Delta^{(i+1)}$, suppose that $v \in F \Delta^{(i+1)}$ is in the kernel of $\delta_{i}$. So $v=$ $\sum_{x \in X} \alpha_{x} x$ for some finite $X \subseteq \Delta^{(i+1)}$ and $\alpha_{x} \in F$. Let $X_{1} \subseteq \Delta$ consist of all the entries from the $p$-sequences in $X$ and let $a \in p\left(X_{1}\right)$. Then for each $x=\left(x_{1}, \ldots, x_{i+1}\right) \in X$, the $(i+2)$-tuple $(x, a)$ is a $p$ sequence. Let $w=\sum_{x \in X} \alpha_{x}(x, a)$. Thus $w \in F \Delta^{(i+2)}$ and we claim that $\delta_{i+1}(w)= \pm v$, which will give the exactness we require.

Indeed,

$$
\delta_{i+2}(w)=\sum_{x \in X} \alpha_{x} \sum_{k=1}^{i+1}(-1)^{k}\left(x_{1}, \ldots, \hat{x_{k}}, \ldots, x_{i+1}, a\right)+(-1)^{i+2} v .
$$

The $a$ in the final coordinate of the terms in the double sum here is essentially irrelevant: the double sum is computing $\delta_{i+1}(v)$, then appending $a$ to all tuples appearing in it. As $v$ is assumed to be in the kernel of $\delta_{i+1}$, we therefore obtain $\delta_{i+2}(w)=(-1)^{i+2} v$, as required.

(3) As $G$ is transitive on each $\Delta^{(i)}$ the fixed points here just consist of the constant functions. For a constant function $f=\alpha$ in $F^{\Delta^{(i)}}$, we have $d_{i} f\left(x_{1}, \ldots, x_{i+1}\right)=\sum_{k=1}^{i+1}(-1)^{k} \alpha$, which is 0 if $i$ is odd and $-\alpha$ if $i$ is even. Exactness of the given sequence now follows.

As before, we suppose that $\Delta$ is a continuous $G$-space and $p$ is a strong type for $G$ on $\Delta$. We let $\Delta_{n}$ denote the transitive $G$-space $\Delta^{(n)}$. Define $G$-maps $\pi_{i}: \Delta_{i+1} \rightarrow \Delta_{i}$ by $\pi_{i}\left(a_{1}, \ldots, a_{i+1}\right)=\left(a_{1}, \ldots, a_{i}\right)$. This gives a tower $\left(\Delta_{i} ; \pi_{i}\right)$ of transitive, continuous $G$-spaces.

Now, $G$ on $\Delta_{n}$ also has a strong type $p_{n}$ : if $X$ is a finite subset of $\Delta_{n}$ let $X_{1}$ be the elements of $\Delta$ appearing in tuples in $X$, and let $p_{n}(X)$ be the $G_{X}$-orbit which contains $\left(y_{1}, \ldots, y_{n}\right)$ where $y_{1} \in p\left(X_{1}\right)$, $y_{2} \in p\left(X_{1} \cup\left\{y_{1}\right\}\right) \ldots$ It is easy to check that $p_{n}$ is a well-defined strong type on $\Delta_{n}$. Thus we may consider $\Delta_{n}^{(j)}$, the set of $p_{n}$-sequences of length $j$ from $\Delta_{n}$. An element of this is a $j$-tuple of $n$-tuples from our original set $\Delta$, and so can be considered as an $n \cdot j$-tuple of elements of $\Delta$. As such, it is easily seen to be a $p$-sequence of length $n \cdot j$.

Corollary 3.3. Suppose that $\Delta$ is a continuous $G$-space and $p$ is a strong type for $G$ on $\Delta$. Let $\Delta_{i}$ denote the set of p-sequences of length $i$ and $\pi_{i}: \Delta_{i+1} \rightarrow \Delta_{i}$ be given by projection onto the first $i$ coordinates. Suppose that for each $j \in \mathbb{N}$ the towers $\left(\Delta_{i}^{j} ; \pi_{i}^{j}\right)$ are smooth. Then $\mathrm{H}_{c}^{n}(G, F)=0$ for all $n>0$. 
Proof. By the smoothness hypothesis we have an acyclic resolution of $F$ as in (6), so we can use (7) to compute $\mathrm{H}_{c}^{*}(G, F)$. But Lemma 3.2 (1) gives that, for fixed $i$, the sequence formed from the $\left(F^{\Delta_{i}^{n}}\right)^{G}$ is exact (using the strong types $p_{i}$ on $\Delta_{i}$ ), hence all homology groups on the right hand side in $(7)$ are zero (for $n \geq 1$ ).

In practice the smoothness condition in this result is awkward to verify, so we give a slightly different approach using parts (2) and (3) of Lemma 3.2. Supose $p$ is a strong type for $G$ on $\Delta$ and use the notation $p_{i}, \Delta_{i}^{(j)}$ given previously. As above, we have a tower of continuous $G$-spaces $\left(\Delta_{i} ; \pi_{i}\right)$ and this gives, for each $j$, a tower $\left(\Delta_{i}^{(j)} ; \pi_{i}^{j}\right)$.

We say that the strong type $p$ is smooth if for every $p$-sequence $a$ and open subgroup $U \leq G$ there is a $p$-sequence $b$ extending $a$ with $G_{b} \leq U$. This terminology is justified by:

LEMMA 3.4. If $p$ is a smooth strong type for $G$ on $\Delta$, then for each $j \in \mathbb{N}$ the tower $\left(\Delta_{i}^{(j)} ; \pi_{i}^{j}\right)$ is smooth.

Proof. As each $\Delta_{i}^{(j)}$ is a transitive $G$-space, we have to show that for every open subgroup $U \leq G$ and $a=\left(a_{1}, \ldots, a_{j}\right) \in \Delta_{i}^{(j)}$, there is $k \geq i$ and $b=\left(b_{1}, \ldots, b_{j}\right) \in \Delta_{k}^{(j)}$ with $b_{r}$ extending $a_{r}$ (for $\left.r=1, \ldots, j\right)$ and $G_{b} \leq U$ (note that this is precisely our smoothness assumption on $p$ in the case $j=1$ ). Now, $a$ can be considered as a $p$-sequence (of length $i \cdot j)$, so by the smoothness of $p$ there is a $p$-sequence $d=(a, c)$ extending it, with $G_{d} \leq U$. Here $c$ is a tuple consisting of the extra coordinates needed to extend the $p$-sequence $a$ to $d$.

Let $k$ be $i$ plus the length of $c$. We show that there is some $b=$ $\left(b_{1}, \ldots, b_{j}\right) \in \Delta_{k}^{(j)}$ with $b_{r}$ extending $a_{r}$ for $r=1, \ldots, j-1$, and $b_{j}=$ $\left(a_{j}, c\right)$. Thus in particular, $G_{b} \leq G_{d} \leq U$. Take any $b^{\prime}=\left(b_{1}^{\prime}, \ldots, b_{j}^{\prime}\right) \in$ $\Delta_{k}^{(j)}$. As $d$ is a $p$-sequence, there is $g \in G$ with $g a_{r}$ an initial segment of $b_{r}^{\prime}$ for $r=1, \ldots, j-1$ and $g\left(a_{j} c\right)=b_{j}^{\prime}$. So we may take $b_{r}=g^{-1}\left(b_{r}^{\prime}\right)$ for $r=1, \ldots, j-1$.

TheOrem 3.5. Suppose $\Delta$ is a continuous $G$-space and $p$ is a smooth strong type for $G$ on $\Delta$.

(1) We have $\mathrm{H}_{c}^{n}(G, F)=0$ for all $n>0$.

(2) For all $m \geq 1$, we have $\mathrm{H}_{c}^{n}\left(G, F^{\Delta_{m}}\right)=0$ for all $n>0$.

Proof. (1) Let $M_{j}$ be the weakly profinite module $\lim _{i} \Delta_{i}^{(j)}$. By Lemma $3.2(2)$, the chain complex

$$
0 \rightarrow F \rightarrow M_{1} \stackrel{d_{1}}{\rightarrow} M_{2} \stackrel{d_{2}}{\rightarrow} M_{3} \stackrel{d_{3}}{\rightarrow} \cdots
$$


is exact. Moreover, by Lemmas 3.4 and 3.1, each $M_{j}$ is acyclic for bounded cohomology. Thus (as with equation (7)) we can compute the cohomology of $G$ on $F$ by taking the homology of the complex of $G$-fixed points of the above resolution. But by Lemma $3.2(3)$, this is zero.

(2) Let $b \in \Delta_{m}$. Define $p^{\prime}(Y)=p(Y b)$, for $Y$ a finite subset of $\Delta$. This is a strong type for $G_{b}$ on $\Delta$ and smoothness of $p$ implies smoothness of $p^{\prime}$. So $\mathrm{H}_{c}^{n}\left(G_{b}, F\right)=0$ for all $n>0$, by part (1). The result then follows from Shapiro's Lemma.

We now recall, from the Introduction, the category $\mathbf{F}_{G}$ whose objects are the closed submodules of finite direct sums of the various $A \Uparrow_{U}^{G}$, where $U$ is an open subgroup of $G$ and $A$ is a finite abelian group (with trivial $G$-action). This is an additive category, but in general, however, it is not clear that $\mathbf{F}_{G}$ will be closed under quotients (by closed submodules). When it is, $\mathbf{F}_{G}$ is an abelian category and in the presence of a smooth strong type, it has enough acyclics for continuous cohomology:

Corollary 3.6. Suppose there is a continuous $G$-space $\Delta$ on which there is a smooth strong type $p$. If $\mathbf{F}_{G}$ is closed under quotients then $\mathbf{F}_{G}$ and $\mathbf{P}_{G}$ are abelian categories with enough acyclics and $\mathrm{H}_{c}^{n}(G, M)$ is finite for all $n$ and $M \in \mathbf{F}_{G}$.

Proof. By the above remarks, $\mathbf{F}_{G}$ is an abelian category. By definition, any $M \in \mathbf{F}_{G}$ embeds into a finite direct sum of coinduced modules $A \Uparrow_{H}^{G}$, and any one of these embeds into some $F^{\Delta_{m}}$ (in the notation of Theorem 3.5). But these are acyclic (by Theorem 3.5).

The finiteness claim follows by dimension shifting and the fact that $\mathrm{H}_{c}^{0}\left(G, F^{\Delta_{m}}\right)=F$, since $G$ is transitive on $\Delta_{m}$.

It remains to show that $\mathbf{P}_{G}$ also has enough acyclics. First, note that any element of $\mathbf{P}_{G}$ can be embedded into a direct product of elements of $\mathbf{F}_{G}$. One way to see this is to work with the Pontryagin duals: as mentioned in the Introduction, the duals of elements of $\mathbf{P}_{G}$ are the discrete, continuous torsion $G$-modules, and duals of elements of $\mathbf{F}_{G}$ are the finitely generated ones.

Thus any element of $\mathbf{P}_{G}$ can be embedded into a direct product of acyclics which lie in $\mathbf{F}_{G}$ : but this is an acyclic in $\mathbf{P}_{G}$.

Corollary 3.7. If $G$ is one of the following permutation groups, then $\mathbf{F}_{G}$ satisfies the conclusions of Corollary 3.6.

(1) The symmetric group acting on $\Omega$.

(2) An infinite dimensional general linear group over a finite field acting on its natural module. 
(3) The group of isometries of a countable dimensional classical space: a vector space $V$ over a finite field equipped with a symplectic, unitary or non-degenerate quadratic form.

Proof. In each case the coinduced modules $F \Uparrow_{U}^{G}$ satisfy the descending chain condition on closed submodules. The same is therefore true for any element of $\mathbf{F}_{G}$, and it then follows that $\mathbf{F}_{G}$ is closed under quotients. (See 7.2-7.7 in [6] for this, phrased in rather different language). So we need to exhibit a smooth strong type in each case.

(1) Take $p(X)=\Omega \backslash X$.

(2) Take $p(X)$ to be the vectors independent from $X$.

(3) For the orthogonal spaces (not characteristic 2) and for the unitary spaces we take $p(\emptyset)=\{v \in V \mid(v, v)=1\}$ and $p(X)=$ $p(\emptyset) \cap\left(X^{\perp} \backslash\langle X\rangle\right)$ (where $(.,$.$) is the bilinear form). Thus p$-sequences are orthonormal sequences, and this gives a smooth strong type.

For the symplectic spaces we consider instead $G$ acting on an orbit $\Delta$ of tuples which enumerate hyperbolic planes (that is, non-degenerate 2 -spaces). This permutation action is faithful and gives rise to the same topology on $G$ as its action on $V$. If $X$ is a finite subset of $\Delta$ let $p(X)$ consist of (suitably enumerated) hyperbolic planes in $\langle X\rangle^{\perp}$. By Witt's theorem this is a single $G_{X}$-orbit, and $p$ is therefore a strong type. But any finite dimensional subspace is contained in a non-degenerate one, which is the orthogonal sum of hyperbolic planes. So $p$ is smooth.

A similar argument handles the case of the orthogonal spaces in characteristic 2 .

We conclude with some remarks about an alternative approach to the continuous cohomology groups in the case where the conclusions of Corollary 3.6 apply. So suppose $G$ is a permutation group and there is a smooth strong type for $G$ on some continuous $G$-space. Let $k$ be either a finite field or the ring $\mathbb{Z} / m \mathbb{Z}$ (for some $m \in \mathbb{N}$ ). Let $\mathbf{P}_{G}[k], \mathbf{F}_{G}[k]$ denote respectively the categories of profinite $k G$-modules with continuous $G$-action and those whose duals are finitely generated; morphisms are continuous $k G$-homomorphisms in each case. Let $\mathbf{C}$ denote one of these categories. Then, the arguments given previously apply to show that $\mathbf{C}$ is an abelian category with enough acyclics for continuous cohomology (and also that the groups $\mathrm{H}_{c}^{*}(G, M)$ are finite if $\left.M \in \mathbf{F}_{G}[k]\right)$.

For $M, N \in \mathbf{C}$ and $n \geq 1$ we can define (Yoneda) Ext-groups $\operatorname{Ext}_{\mathbf{C}}^{n}(M, N)$ in terms of equivalence classes of $n$-extensions

$$
0 \rightarrow N \rightarrow E_{n} \rightarrow \cdots \rightarrow E_{1} \rightarrow M \rightarrow 0
$$


in $\mathbf{C}$ (see [3] for details); we also let $\operatorname{Ext}_{\mathbf{C}}^{0}(M, N)=\operatorname{Hom}_{\mathbf{C}}(M, N)$. We claim that $\operatorname{Ext}_{\mathbf{C}}^{n}(k, N) \cong \mathrm{H}_{c}^{n}(G, N)$, for $N \in \mathbf{C}$.

In fact, the argument is exactly as in ([12], page 86$)$, but we give a few more details. The sequences of covariant functors $\operatorname{Ext}_{\mathbf{C}}^{n}(k,-)$ and $\mathrm{H}_{c}^{n}(G,-)$ are exact connected sequences of functors on $\mathbf{C}$ (in the terminology of $[4])$. Moreover, by the dual version of ([4], Proposition $4.2)$, they are both universal: for the first sequence, this follows as in

([4], Proposition 4.3); for the second it follows from the existence of enough acyclics. Thus, as the sequences agree at the first term $n=0$, they are naturally equivalent.

\section{References}

[1] G Ahlbrandt, M Ziegler, "What's so special about $(\mathbb{Z} / 4 \mathbb{Z})^{\omega}$ ?", Archive for Math. Logic 31 (1991), 115-132.

[2] R Brown, P J Higgins, S A Morris, "Countable products and sums of lines and squares: their closed subgroups, quotients and duality properties", Math. Proc. Camb. Phil. Soc. 78 (1975), 19-32.

[3] D Buchsbaum, "A note on homology in categories", Ann Math 69 (1959), 199-201.

[4] D Buchsbaum, "Satellites and universal functors", Ann Math 71 (1960), 6674.

[5] D M Evans, "Finite covers with finite kernels", Ann Pure App Logic 88 (1997), 109-147.

[6] D M Evans, "Computation of first cohomology groups of finite covers", J Algebra 193 (1997), 214-238.

[7] D M Evans, A A Ivanov, H D Macpherson, "Finite covers", in Model Theory of Groups and Automorphism Groups, ed. David M Evans, London Mathematical Society Lecture Notes 244, Cambridge Univ Press, Cambridge, 1997.

[8] A Guichardet, Cohomologie des groupes topologiques et des algèbres de Lie. Cedic/Fernand Nathan, Paris, 1980.

[9] W Hodges, A Pillay, "Cohomology of structures and some problems of Ahlbrandt and Ziegler", J. London Math. Soc. (2) 50 (1994), 1-16.

[10] P J Higgins, "Coproducts of topological abelian groups", J Algebra 44 (1977), 152-159.

[11] J-P Serre, Galois Cohomology. Springer-Verlag, Berlin, 1991.

[12] D Wigner, "Algebraic cohomology of topological groups", Trans AMS 178 (1973), 83-93.

School of Maths, Univ of East Anglia, Norwich NR4 7TJ, England

E-mail address: d.evans@uea.ac.uk

Dept of Math, Univ of Toledo, Toledo OH 43606, USA

E-mail address: paul.hewitt@utoledo.edu 